\title{
LA CATASTROFE DEL BARRANCO DE ARÁS (7/8/1996): PROCESOS NATURALES E HIPÓTESIS EXPLICATIVA ${ }^{1}$
}

\author{
Luis CANCER POMAR \\ Departamento de Geografía y Ordenación del Territorio \\ Universidad de Zaragoza
}

\begin{abstract}
Resumen: Los efectos catastróficos de la crecida de caudales del barranco de Arás el 7 de agosto de 1996 fueron consecuencia de unas precipitaciones excepcionalmente intensas y del gran aporte de sólidos al cauce, procedentes de unas laderas enmarcantes muy erosionables. En el artículo se calculan los caudales posibles bajo distintas hipótesis y se analizan las características geomorfológicas de la cuenca, especialmente de los depósitos detríticos que actuaron de área fuente de los sedimentos transportados por el barranco. Además de los procesos naturales actuantes, determinadas actuaciones antrópicas de gestión del medio han contribuido a provocar una catástrofe de gran magnitud.
\end{abstract}

Palabras clave: Precipitación, intensidad, crecida, erosión, procesos, riesgos, catástrofe, gestión del medio.

\begin{abstract}
Catastrophic effects in the Aras gully's flood exceptionally intense rainfall and the great contribution of solids to riverbed, proceed from hillsides very erosives. This article calculates possible flows from different hypothesis and analyses geomorphologic characteristics of the catchment area, especially detritus deposits operated as source area of the sediments transported along the gully. Besides natural operation processes, the anthrophic actions determined in the management of the environment have contributed to the origination of catastrophe of with an enormous magnitud.
\end{abstract}

Key words: Rainfall, intensity, flood, erosion, processes, risks, catastrophe, environment management.

\section{INTRODUCION}

En la tarde del 7 de agosto de 1996 se produjo sobre la alta cuenca del río Gállego, en el Pirineo central, una precipitación de agua y granizo de gran intensidad.

1 Artículo entregado a la redacción de la revista Geographicalia el 2 de septiembre de 1996. 
Las consecuencias se dejaron sentir especialmente en la cuenca del barranco de Arás, cercano a la población de Biescas, en donde el desbordamiento de su cauce arrasó un camping situado en su cono de deyección, causando 87 muertos e ingentes pérdidas y destrozos materiales. En este artículo se intenta establecer las razones explicativas de tal catástrofe, atendiendo tanto a los datos que se tienen acerca del funcionamiento de la cuenca como a las pistas proporcionadas por el trabajo de campo in situ llevado a cabo durante los días inmediatamente posteriores a la tragedia.

\section{DATOS DE PRECIPITACION}

La tormenta comenzó a las 17,30 h., aumentando su intensidad de forma muy acusada desde las $18,45 \mathrm{~h}$. hasta las $19,30 \mathrm{~h}$. A esta última hora se produjo el arrasamiento del Camping "Las Nieves". Luego, continuó lloviendo, aunque más débilmente, hasta las $24 \mathrm{~h}$.

La única estación pluviométrica de la cuenca del barranco de Arás se sitúa en Aso de Sobremonte, aguas arriba del camping, a unos $4 \mathrm{~km}$. en línea recta. En este punto el registro marcó $170 \mathrm{~mm}$ en un período de $2 \mathrm{~h}$. 15', entre las 17,30 y las 19,45 aproximadamente. Pero esta cifra no indica la totalidad de agua caída, ya que el pluviómetro se había desbordado antes de que fuera anotado el registro. Además, tal como se ha comentado líneas arriba, la intensidad de la precipitación no fue homogénea en el tiempo, ya que el mayor volumen se produjo en un período de no más de 30 ó 45 minutos $^{2}$.

Esta precipitación extraordinariamente intensa se circunscribió a una zona de muy reducidas dimensiones, ya que los datos de estaciones cercanas, aunque indican fuertes lluvias, son muy inferiores a los de Aso (salvo en el caso de Biescas -Estación Meteorológica "Central 1"-). Valgan como ejemplo los siguientes, referidos al día 7 de agosto de $1996\left(\mathrm{en} \mathrm{mm} / \mathrm{m}^{2}\right)^{3}$ :

$\begin{array}{lr}\text { Sallent de Gállego: } & 46,0 \\ \text { El Pueyo de Jaca: } & 51,0 \\ \text { Biescas (Central 1): } & 160,0 \\ \text { Sabiñánigo: } & 37,5\end{array}$

\footnotetext{
2 El pluviómetro de Aso de Sobremonte no cuenta con banda pluviográfica, por lo que no registra el dato de la intensidad horaria. Los datos aproximados aquí aportados proceden de las informaciones facilitadas por los propios vecinos de este núcleo.

3 Proporcionados por el Instituto Nacional de Meteorología (Centro Territorial de Aragón, La Rioja y Navarra).
} 
En estas estaciones, sólo en Sallent y Biescas fue el día 7 el de precipitación máxima durante el mes de agosto. En El Pueyo de Jaca y Sabiñánigo fueron otras las fechas de máxima precipitación.

La tromba de agua tuvo, pues, una localización muy concreta, limitada al sector norte de la denominada "Ribera de Biescas", inmediatamente al Sur del valle de Tena. Incluso en el interior de la cuenca del Arás, ciertos indicios permiten aventurar que tampoco las precipitaciones fueron homogéneas en el espacio, resultando especialmente abundantes en la pequeña subcuenca de Betés, como luego veremos.

La precipitación registrada en Aso, de $170 \mathrm{~mm}$, debe ser comparada con eventos anteriores y predicciones estadísticas para calibrar realmente su magnitud. Ante la falta de datos referidos exactamente a la zona que nos interesa, hay que acudir a 1 a vecina estación de Biescas, en donde los cálculos de precipitaciones máximas diarias para determinados períodos de retorno ofrecen los siguientes valores:

$\begin{array}{ccc}\text { Período de retorno (años) } & \text { Pp. }(\mathrm{mm})^{*} & \operatorname{Pp}(\mathrm{mm})^{* *} \\ 5 & 89 & 84,3 \\ 10 & 106 & 102,8 \\ 20 & 122 & 120,5 \\ 50 & 143 & 143,3 \\ 100 & 159 & 160,2 \\ 200 & 175 & - \\ 500 & 196 & -\end{array}$

* Datos de MARÍN JAIME (1987).

** Datos del ICONA (1979).

Como puede apreciarse, ambas fuentes coinciden sensiblemente. El ICONA no proporciona datos de períodos de retorno superiores a 100 años, pero sí Marín Jaime, y de ellos puede deducirse que la precipitación registrada en Biescas (160 mm) coincide exactamente con esperada para un período de retorno de 100 años, mientras que la de Aso coincidiría con la esperada en Biescas para un período de retorno de entre 100 y 200 años, más próxima a esta última previsión 4 .

Dado que los datos aportados se refieren a una estación -Biescas- ubicada fuera de la cuenca del barranco de Arás, hay que señalar que se encuentra muy cercana a Aso, a tan sólo $3,5 \mathrm{~km}$ en línea recta, y a sólo $1 \mathrm{~km}$ del sector inferior de esta cuenca. Ambas

\footnotetext{
4 A modo de muestra de datos registados en Biescas, la precipitación máxima en 24 horas entre 1950 y 1980 fue de 118,6 mm el 4 de marzo de 1959 (CUADRAT PRATS, 1981, p. 260).
} 
poblaciones se encuentran a idéntica latitud, aunque a altitud diferente: $1264 \mathrm{~m}$ de Aso frente a $875 \mathrm{~m}$ de Biescas. Razones topográficas y de exposición a las advecciones húmedas explican que esa variación altimétrica no se refleje en los valores de precipitación anual (1214 mm en Aso y $1237 \mathrm{~mm}$ en Biescas). Sin embargo, los estudios realizados por MARÍN JAIME (1987) indican que las precipitaciones máximas en 24 horas son mayores en las estaciones situadas a más altitud, de manera que la precipitación registrada en Aso el 7 de agosto de 1996 coincidiría con la esperada para un período de retorno ligeramente inferior al de Biescas.

\section{CAUDAL ESTIMADO}

Para establecer un volumen aproximado del caudal que circuló por el barranco, se va a partir de datos conocidos y contrastados, pero que no reflejan totalmente la realidad de lo ocurrido en esta cuenca, tal como se explica a continuación:

- El dato de precipitaciones utilizado es de $170 \mathrm{~mm} / \mathrm{m}^{2}$ en $135 \mathrm{~min}$., pero tal como se ha indicado líneas arriba, fue superior, aunque se desconoce en qué medida.

- El dato de coeficiente de escorrentía de esta cuenca es una incógnita, y a que no dispone de estación de aforo que permita realizar los cálculos pertinentes. Hay que acudir, por aproximación, a otros sectores de la cuenca del Gállego que sí se hallan equipados con aforos. El más cercano es de la presa de Búbal, que refleja el comportamiento hidrológico de la práctica totalidad del valle de Tena. Los estudios realizados indican un valor medio anual de 0,82, casi coincidente con el valor medio del mes de agosto, de 0,83 (GARCÍA RUIZ, PUIGDEFABREGAS y CREUS, 1986). Sin embargo, las características físicas del valle de Tena son muy distintas a las existentes en la cuenca del Arás (mayores pendientes y menor cubierta vegetal en Tena, litologías diferentes, etc. -ver CANCER POMAR, 1995-) y permiten suponer, con poco margen de error, un mayor coeficiente en Tena que en Arás. MARÍN JAIME (1987), por su parte, calcula para el sector de la cuenca del Gállego comprendido entre los aforos de Búbal y Anzánigo (en el sector septentrional de este tramo se encuentra el barranco de Arás) un coeficiente medio anual de 0,37. Este valor tampoco puede ser representativo del comportamiento de la zona que nos interesa, ya que en buena parte de este sector del Gállego las condiciones físicas son diferentes a las de Arás, siendo previsible que en esta última cuenca el coeficiente sea mayor ${ }^{5}$.

5 Aguas abajo de Biescas, las características topográficas -pendientes inferiores a las de la cuenca alta del Gállego, numerosos sectores llanos- hacen previsible un coeficiente de escorrentía inferior al de Arás. 
Por último, el mismo autor calcula un valor de 0,52 para la cuenca del Gállego desde su cabecera hasta Anzánigo, que refleja el valor medio de un espacio con grandes contrastes internos.

Aun cuando posiblemente este último coeficiente sea el más aproximado al de Arás, se trata en todos los casos de valores que indican comportamientos hidrológicos medios, muy diferentes a los existentes bajo condiciones extremas, como la que nos ocupa.

En los momentos puntuales de precipitaciones de gran intensidad, el coeficiente de escorrentía aumenta y se sitúa próximo al valor 1, a los cual contribuyen varios factores: tras los primeros minutos de precipitación, una vez que el terreno se $\mathrm{ha}$ saturado de agua, la retención de este líquido en superficie es prácticamente nula; en las cuencas de pendientes medias y altas -como la de Arás- la tasa de infiltración se ve muy mermada, ante la gran velocidad de la escorrentía superficial; las pérdidas por evapotranspiración e interceptación resultan muy inferiores a las producidas bajo condiciones de precipitación poco intensa.

En los cálculos presentados en la tabla anexa se muestran los caudales del barranco de Arás bajo distintos supuestos, desde el coeficiente de escorrentía 1 (a modo de dato teórico, ya que en sentido estricto nunca se produce en la realidad) hasta el 0,52, pasando por el 0,82 . Posiblemente los valores comprendidos entre 0,82 y 1 sean los que más se aproximen al comportamiento de la cuenca bajo estas condiciones excepcionales. También se han introducido dos supuestos respecto a la distribución temporal de las precipitaciones, considerando en el primero un reparto homogéneo durante $2 \mathrm{~h} .15 \mathrm{~min}$. y en el segundo, suponiendo que en los 45 minutos de mayor intensidad se registrara el $80 \%$ de la precipitación total (es decir, $136 \mathrm{~mm} / \mathrm{m}^{2}$ ).

Los datos presentados en la tabla se refieren al caudal supuesto del barranco de Arás en su tamo inferior (en el cono de deyección), por lo que reflejan las precipitaciones caídas en toda su cuenca hidrográfica, de una superficie de $19,4 \mathrm{~km}^{2}$.

Cualquiera de los datos presentados suponen escorrentías homogéneamente repartidas durante los períodos considerados. Se trata, por lo tanto, de caudales medios durante 135 ó 45 minutos. En función de las precisiones anteriores, las cifras supuestas de caudal oscilarían entre 330 y más de $900 \mathrm{~m}^{3} / \mathrm{sg}$. Sin embargo, según las informaciones proporcionadas por testigos presenciales e incorporando las impresiones recibidas en el trabajo de campo, puede indicarse la posibilidad muy probable de que se produjeran momentos con puntas de caudal mayores, por razones que más adelante se explican. 


\section{Caudales estimados bajo diferentes supuestos (en $\mathrm{m}^{3} / \mathrm{sg}$ )}

$$
\begin{array}{lll}
\text { C.E. }=1 & \text { C.E. }=0,82 & \text { C.E. }=0,52
\end{array}
$$

$170 \mathrm{~mm} / 135^{\prime}$

407

333

211

$136 \mathrm{~mm} / 45^{\prime}$

978

801

508

Pero la canalización de Arás no tuvo sección suficiente para desaguar el caudal del barranco, lo cual se pudo constatar inequívocamente en numerosos puntos de 1 a sección inferior de las laderas enmarcantes del talweg, en los trabajos de campo llevados a cabo tras la tragedia. Por ejemplo, en los prados situados debajo del núcleo de Yosa de Sobremonte, se apreció el nivel exacto al que llegaron las aguas desbordadas, por el aplastamiento de la hierba y la deposición de una guirnalda lateral longitudinal de pequeños materiales, ramas sobre todo. En algunos tramos, las aguas alcanzaron una altura de entre 4 y $10 \mathrm{~m}$ por encima de la canalización.

Los barrancos afluentes también se desbordaron. Quizá el punto en el que mejor se aprecia este hecho sea el puente sobre el barranco de Betés -afluente por la izquierda del Arás-, en la carretera de Aso. La barrera guardarraíles está totalmente arrancada, lo cual indica que el agua pasó por encima. La plataforma del puente está a unos $7 \mathrm{~m}$ de altura con respecto al fondo del barranco. Los pilares quedaron descalzados, como consecuencia de la remoción de materiales.

\section{CARACTERISTICAS GEOMORFOLOGICAS}

Explicados someramente los datos de precipitación y de caudales supuestos, es necesario referirse a las características geomorfológicas de la zona. El desbordamiento que sufrió el barranco de Arás no parece que pueda explicarse únicamente por el volumen de precipitaciones. A este factor hay que añadir otro de tipo geomorfológico, de manera que dos modalidades de riesgos naturales se habrían unido para provocar un efecto catastrófico multiplicador.

La cuenca del barranco de Arás se instala en los relieves del flysch eoceno adosado a las Sierras Interiores prepirenaicas, aunque extiende su cabecera, en el sector de las Peñas de Aso, hasta el borde meridional de estas sierras. Pertenece a 1 tramo de la cuenca del Gállego conocido como la Ribera de Biescas, situándose en la margen derecha de su sector septentrional. 


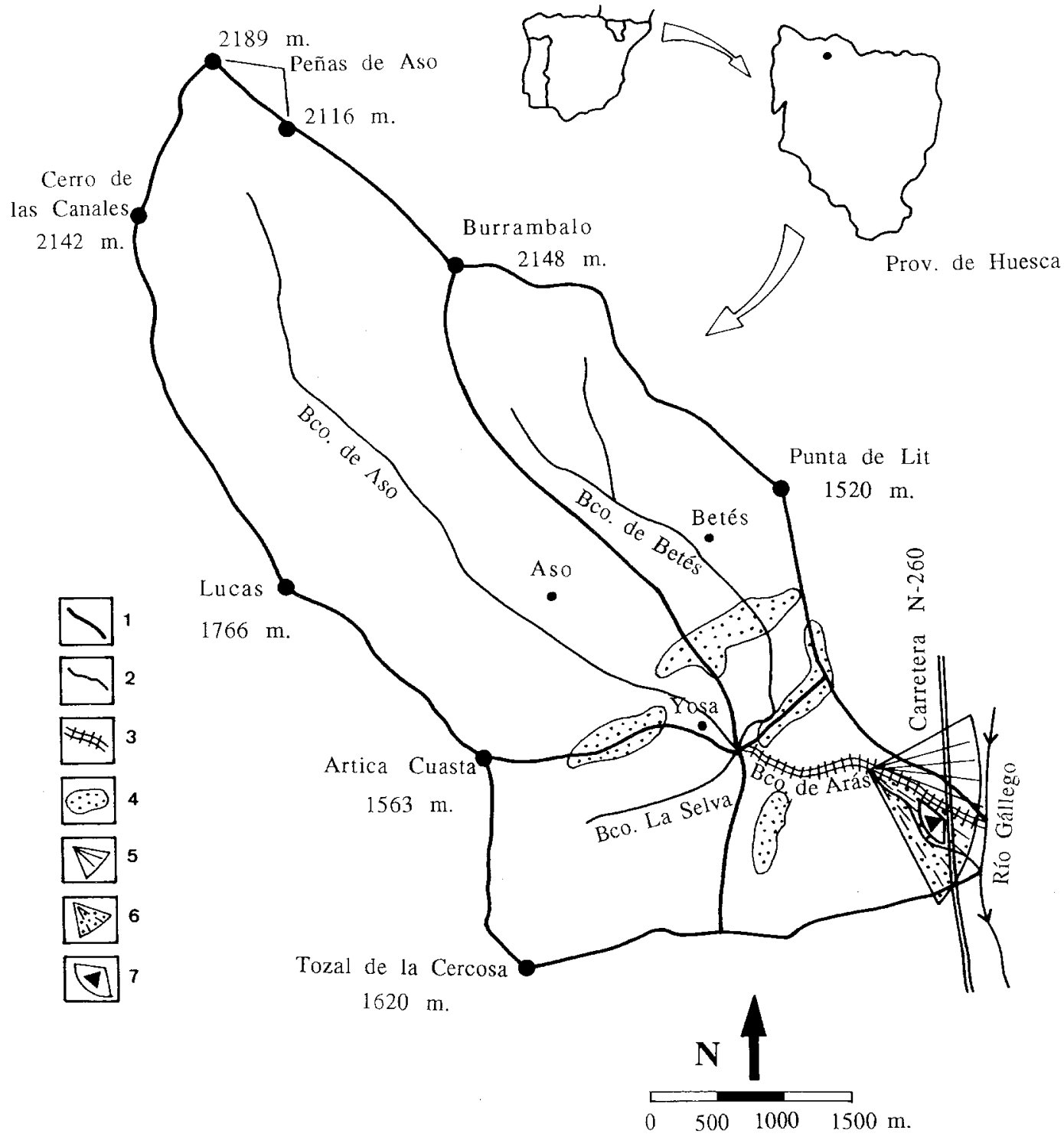

Figura 1.- Area de estudio.

1.- Divisoria de cuencas hidrográficas. 2.- Cursos fluviales. 3.- Tramo canalizado del bco. de Arás. 4.- Cordones morrénicos. 5.- Cono de deyección no funcional. 6.- Cono de deyección funcional. 7.- Camping. 
El barranco de Arás nace al pie de las citadas Peñas de Aso (2189 m) y desemboca en el Gállego, aguas abajo de Biescas, a $840 \mathrm{~m}$. La cuenca de este barranco denominada en la Ribera de Biescas con la expresión de "Sobremonte"- está conformada por tres pequeñas subcuencas de cabecera. Son las siguientes:

- Cuenca del barranco de Aso. Es la más extensa $\left(10,66 \mathrm{~km}^{2}\right)$ y la ubicada en posición central. De sentido NW-SE, en ella se encuentra situado el núcleo de Aso de Sobremonte.

- Cuenca del barranco de La Selva, afluente del Aso por la derecha. De sentido WSW-ENE, drena el sector situado inmediatamente al Sur de Yosa de Sobremonte. Su extensión es de sólo $2,67 \mathrm{~km}^{2}$.

- Cuenca del barranco de Betés, afluente del Aso por la izquierda, de sentido NW-SE y con 4,26 km². Aquí se ubica el núcleo de Betés.

A partir de la confluencia de las dos últimas en el barranco principal -que se produce en el mismo punto, una enfrente de la otra, aguas abajo de Yosa- el curso resultante adopta la denominación de Barranco de Arás. La superficie de la cuenca aguas abajo de esta confluencia es de $1,84 \mathrm{~km}^{2}$.

Las sierras flyschoides que enmarcan esta cuenca (Burrambalo: $2148 \mathrm{~m}$. ; Cerro de la Canales: $2142 \mathrm{~m}$. ; Lucas: $1766 \mathrm{~m}$. ; Limes: $1465 \mathrm{~m}$. , etc.) se caracterizan por un modelado indefinido de colinas y alineaciones de cumbres bastante redondeadas, con laderas muy homogéneas en las que son abundantes los deslizamientos en masa, así como arroyamiento superficial en surcos y cabeceras activas (GARCÍA RUIZ y PUIGDEFABREGAS, 1982). El sistema morfogenético se halla además influido decisivamente por la existencia de varios conjuntos morrénicos sobre el flysch, y por los mecanismos derivados de la morfogénesis fluvial postglaciar.

Estas morrenas fueron depositadas por el glaciar del Gállego, que en el momento de máximo glaciar desbordó la artesa principal y se introdujo por los valles laterales, generando las correspondientes lenguas y depositando arcos morrénicos de obturación (Figura 1). Varios de estos conjuntos morrénicos son atravesados por los cursos fluviales existentes (barrancos de Aso, Betés y Yosa), tomando muchos sedimentos de variado calibre por socavamiento basal. Por otra parte, la cuenca del barranco de Arás se halla suspendida sobre el valle del Gállego, por derivar de un valle colgado con respecto a 1 valle glaciar principal. Tal como señala GÓMEZ VILLAR (1996), el paso por las morrenas y la fuerte pendiente del canal poco antes de llegar a la Ribera de Biescas, explican el gran potencial erosivo y de transporte del barranco y la formación del cono 
de deyección al entrar en contacto con la llanura de la Ribera de Biescas. Este cono es funcional en su sector derecho, mientras que el izquierdo es no funcional.

En bastantes sectores de las laderas, pero sobre todo en el contacto entre las vertientes y los fondos de valle, aparecen depósitos de ladera relacionados con momentos fríos coetáneos al retroceso del máximo glaciar (SERRANO CAÑADAS, 1991). Abundantes materiales coluviales procedentes de estos depósitos, acusadamente angulosos, tapizan los talwegs de los barrancos, sobre todo aguas arriba de las morrenas. Su débil índice de rodamiento señala la importante actividad del proceso de aportes de materiales desde las laderas hasta los fondos de valle.

Como consecuencia de esta evolución geomorfológica, la morfología actual es de morrenas derrubiadas en donde, además de los depósitos glaciares, se han acumulado materiales de diferente procedencia, coluviales, fluviales y lacustres, estos últimos a partir de la formación de cubetas de obturación. Este carácter poligénico de los materiales explica su variedad facial y su heterometría; grandes bloques de cuarcitas, pizarras y en menor medida de granito, envueltos con una matriz arcillosa, que incluye clastos calcáreos y areniscosos del flysch eoceno, tal y como señalan PELLICER CORELLANO, CANCER POMAR y ECHEVERRÍA ARNEDO (1994).

\section{HIPOTESIS EXPLICATIVA}

La causa inicial de la catástrofe fueron las intensas precipitaciones, que produjeron unos caudales anormalmente altos. Al actuar la escorrentía sobre materiales detríticos fácilmente erosionables, gran cantidad de sólidos heterométricos fueron arrastrados por las aguas, aumentando la densidad y el poder destructivo del flujo. Pero, además de estos procesos naturales, resulta necesario introducir determinadas informaciones referidas a aspectos antrópicos de gestión del medio natural, imprescindibles para esbozar las razones de la catástrofe.

\section{Barranco de Aso}

Comenzando por la cuenca principal, la del barranco de Aso, de la impresión recibida en los trabajos de campo y de las informaciones proporcionadas por sus habitantes puede deducirse que los destrozos no son de gran importancia. El casco urbano no ha resultado dañado, aunque varios caminos de su entorno, que actuaron a modo de canales de escorrentía y fueron incididos linealmente por las aguas, han quedado totalmente inservibles. En varios puntos de las laderas ocupadas por prados, se han generado coladas de barro de pequeño tamaño, métricas. Respecto al talweg del barranco, su actual morfología, totalmente antropizada, es la consecuencia de los 
movimientos de tierras efectuados durante la primavera de 1996. En febrero y diciembre de 1995, se produjeron en la zona precipitaciones muy intensas y crecidas de caudales que arrastraron abundantes materiales por el cauce del Aso y destruyeron varios pequeños puentes de acceso a las fincas ${ }^{6}$. En los meses posteriores se acometieron obras de encauzamiento del barranco, procediendo a acumular los materiales aluviales en las orillas y configurando un canal de desagüe rectilíneo. Esta modificación drástica de la geomorfología fluvial ha provocado alteraciones en las condiciones del flujo, discurriendo ahora el agua a más velocidad. Desde el punto de vista de la dinámica fluvial, la conversión del anterior cauce (con acumulaciones irregulares y cambiantes de gravas y bolos que impedían el discurrir recto del barranco) en un canal rectilíneo con ausencia de discontinuidades y de barreras, con un fondo de topografía aplanada y bastante homogénea, ha provocado la disminución del rozamiento basal y lateral, de forma que la velocidad del agua aumenta, fenómeno que se ve acrecentado por el incremento efectivo de la pendiente longitudinal del curso (se salva el mismo desnivel en un recorrido menor). La desaparición de las irregularidades del taliveg provoca la inexistencia de barreras de choque que dispersen la energía del agua, por lo que estas obras de canalización, realizadas para disminuir el riesgo de desbordamientos, desencadenan un efecto no deseado y que puede resultar más pernicioso: el aumento de la energía del flujo, fenómeno en el que el aumento de la velocidad contribuye de forma exponencial $\left(\mathrm{E}=\mathrm{m} \times \mathrm{v}^{2}\right)$.

Aguas abajo de la cubeta de Aso (conformada por depósitos de obturación glaciar), el valle presenta mayor pendiente y un importante estrechamiento del talzueg, al atravesar la morrena, punto en el que se sitúa en puente de acceso a la población de Yosa. El "efecto embudo", acompañado de la elevada velocidad de las aguas, consecuencia tanto del fuerte caudal como de la canalización del cauce, provocaron el arrasamiento de este puente, no tanto como consecuencia del choque directo del flujo contra la estructura, sino debido al socavamiento del punto de anclaje de uno de sus dos pilares de sustentación.

\section{Barranco de Betés}

De los desastres producidos por las precipitaciones y las riadas consiguientes, puede deducirse que la cuenca que registró mayores volúmenes de lluvia fue la de Betés. Aquí los efectos destructivos y arrasadores fueron muy superiores a los de las otras dos subcuencas (Aso y La Selva), algo que se puede apreciar especialmente

\footnotetext{
${ }^{6}$ Estas elevadas precipitaciones, que afectaron a todo el Alto Gállego y a otros valles pirenaicos, estuvicron acompañadas de varios días de temperaturas anormalmente altas que fundieron las reservas de nieve existentes. En estaciones de esquí como Formigal y Panticosa desapareció la innivación, lo mismo que en las cotas altas de la cuenca de Arás. La fusión nival se unió a las fuertes precipitaciones, provocando elevados caudales, que en caso del barranco de Arás estuvieron a punto de desbordar el canal en el cono del deyección.
} 
comparando la situación en que han quedado los cascos urbanos de las tres pequeñas aldeas existentes. En Betés las calles fueron literalmente cubiertas por piedras, que llegaron a alcanzar en varios puntos los $4 \mathrm{~m}$ de altura e incluso derribaron algún edificio ${ }^{7}$. Absolutamente todos los campos del entorno de esta población quedaron arrasados, cubiertos de lodo y piedras. El agua se llevó los campos y las huertas más próximas al cauce, y el talweg de éste ha cambiado intensamente su morfología anterior, tanto por estar totalmente tapizado por bloques y cantos como por sus modificaciones de trazado.

Pero en la cuenca de Betés tampoco se aprecia uniformidad en cuanto a los procesos, siendo éstos de mayor magnitud en la vertiente izquierda del valle que en la derecha. Un ejemplo ilustrativo puede servir para explicar esto: inmediatamente a l NNE de Betés existe un pequeño barranco tan apenas incidido en la ladera, conocido como el barranco del Cagicar. Tan escasa es su importancia, y tan excepcional su funcionamiento como eje de drenaje superficial, que su teórico talweg está ocupado en las cercanías de Betés por un camino, y ya en el interior de este núcleo, es la calle principal la que ocupa la línea de desagüe. Sin embargo, el 7 de agosto de 1996, su habitual falta de actividad dio lugar a una auténtica riada, y a él se debe el aporte de sólidos que arrasó el pueblo, constituido por cantos angulosos de los depósitos coluviales y de los afloramientos de flysch circundantes.

Tanto estas apreciaciones como las observadas aguas abajo, parecen confirmar la idea de que fue en esta pequeña subcuenca donde se registraron las precipitaciones más intensas, concretamente en la vertiente ubicada entre la Punta Burrambalo y la Punta de Lit (figura 1). Pero esta impresión no puede confirmarse fehacientemente, ya que en Betés no existe pluviómetro.

Los aportes de sólidos de esta cuenca, mayores que en las otras, se explicarían fundamentalmente por la mayor intensidad de las precipitaciones, ya que tanto las características geomorfológicas como el tipo de vegetación, cobertura del suelo y valores de pendientes ${ }^{8}$ son muy similares en unas y otras. No obstante, hay que señalar otro factor que podría explicar, aunque sólo parcialmente y con las necesarias reservas, este mayor aporte de sedimentos: las talas efectuadas en la cuenca de Betés durante los años 80. Comparando las fotografías aéreas de 1981 con la situación actual se aprecia que dos zonas han visto reducidas drásticamente sus masas arbóreas: el barranco de Burrambalo, que ocupa el sector occidental de la cuenca, y la vertiente oeste de la Artica del Lugar, en el sector oriental de la cuenca. CANCER POMAR (1995) señala que "el barranco de Burrambalo ha sido talado prácticamente a matarrasa a ambos

\footnotetext{
7 Tres días después de la riada, todavía estaban las palas excavadoras desescombrando este núcleo urbano.

8 La pendiente transversal media de la cuenca del barranco de Betés es del 44,6\% (CANCER POMAR, 1995).
} 
lados del talweg (...). En la Artica del Lugar la explotación, aun siendo intensa, no llega a la matarrasa. Se centra en los pequeños barrancos situados aguas arriba de Betés". Precisamente, estos sectores intensamente talados están tapizados por depósitos coluviales, que han quedado desprovistos de cubierta vegetal protectora, y los cantos angulosos calcáreos y areniscosos procedentes de ellos son los más abundantes en los sedimentos que han invadido el casco urbano de Betés y arrasado sus campos cercanos. Además, la vertiente oeste de la Artica del Lugar está situada justo encima de Betés, y de ella proceden los sedimentos citados.

\section{Barranco de Arás}

En el tramo del barranco de Arás comprendido entre la confluencia de los barrancos de Betés y La Selva y la curva que marca la transición al sector inferior -cono de deyección-, se registra la mayor destrucción del cauce canalizado del Arás, lo cual además de otras razones- permite lanzar la hipótesis de que aquí se debieron concentrar los procesos naturales más violentos. Vamos a intentar descubrir las causas.

El barranco de Arás se halla canalizado desde unos pocos metros más arriba de la desembocadura de los barrancos de Betés y La Selva hasta su desembocadura en el Gállego. La canalización consiste en una sólida obra de sillares de piedra que dibujan gradas, de manera que el perfil del canal se resuelve en una sucesión de escalones. E1 tramo canalizado puede dividirse en dos sectores, de características diferentes. En el sentido de la corriente, el primero comprende desde el inicio de la canalización hasta una curva a la derecha, con una longitud de unos $1.200 \mathrm{~m}$, pendiente longitudinal del $12,5 \%$ y sentido WNW-ESE; la canalización se realizó sobre el cauce antiguo. El segundo va desde la curva hasta la desembocadura en el Gállego, con una longitud de unos $1.150 \mathrm{~m}$., pendiente longitudinal del $8,7 \%$ y sentido NW-SE, atravesando el cono de deyección del barranco; la canalización se trazó a la izquierda del cauce antiguo, y entre ambos cauces, sobre el sector del cono funcional, se levantó el camping "Las Nieves" en 1988, que resultó arrasado por la riada del 7 de agosto de 1996.

El sector inferior es el más conocido, pues resulta totalmente visible desde la carretera de acceso a Biescas y al valle de Tena. Su canalización terminó de construirse en 1926 y, debido a la escasa pendiente del cono de deyecciôn, las gradas presentan saltos débiles, de 2 ó 3 metros. El sector superior es de morfología distinta. La necesaria adaptación a la mayor pendiente longitudinal (es el tramo del barranco situado aguas abajo de las cubetas de obturación glaciar y antes del cono de deyección) obligó a levantar gradas de hasta $10 \mathrm{~m}$ de altura. Además, en muchas de ellas, inmediatamente antes del salto se construyeron diques transversales al flujo, de retención de sólidos y laminación de avenidas, dado el marcado carácter torrencial del barranco y la erosionabilidad de la cuenca. Estos diques dejaban portillos de unos 3 ó 4 
$m$ de ancho para el paso de la escorrentía. Las obras del tramo concluyeron más tarde que en el sector inferior, en la década de los años $40^{9}$.

La sección del canal no es homogénea en toda su longitud. En el sector inferior se trazó un pasillo de $18 \mathrm{~m}$ de ancho, con muros laterales de altura variable según los tramos, no superando ésta 1,20 ó 1,40 m en bastantes zonas, por ejemplo aguas abajo del puente sobre la carretera N-260. En el sector superior la anchura se adapta a las condiciones del cauce natural, pero en general es mayor que en el inferior.

Los episodios de precipitaciones de gran intensidad, relativamente frecuentes en esta cuenca, han provocado desde siempre la caída al canal de grandes bloques procedentes de las morrenas derrubiadas y de abundante material fino. Precisamente uno de los últimos episodios, el acaecido en diciembre de 1995, movilizó abundantes materiales, que quedaron sobre el canal y se sumaron a los preexistentes. Tal como señalan MARTÍNEZ CASTROVIEJO y GARCÍA RUIZ (1990), la gran cantidad de sedimentos acumulados en los cauces asegura la disponibilidad de materiales durante décadas, susceptibles de ser removidos en las crecidas.

La precipitación del 7 de agosto de 1996 movilizó, sin ninguna duda, tal como y a se ha indicado más arriba, gran cantidad de sólidos, tanto procedentes de las laderas como los ya ubicados sobre el propio cauce. Esta evidencia avala la hipótesis de que se produjeran uno o varios taponamientos, a modo de represa temporal de las aguas, aprovechando los diques transversales de las gradas del tramo superior. Tras la riada, en todo el taliveg del Arás, incluso en las gradas más inferiores, a muy pocos metros del rio Gállego, se localizan bloques morrénicos de gran tamaño, que en ocasiones superan los $5 \mathrm{~m}$ de eje mayor y que han sido transportados por el flujo, lo que da idea de su capacidad erosiva y de la competencia del transporte ${ }^{10}$.

En opinión de quien firma este artículo, uno de los puntos clave de este fenómeno se situaría inmediatamente aguas abajo de la desembocadura del barranco de Betés. Observando su taliveg en los metros finales, tras haber atravesado la morrena que delimita la cubeta de Betés, se aprecia la gran cantidad de material morrénico movilizado, con grandes bolos, que se debieron sumar a los materiales coluviales arrastrados en la cuenca alta (a los que ya se ha hecho referencia al comentar 1 a

\footnotetext{
9 La Memoria del año 1912 del Servicio Hidrológico-Forestal (Ministerio de Fomento), que enumera los trabajos de la Sexta División (Cuenca media del Ebro), refiere ya las obras de correción del barranco de Arás, acompanadas de la repoblación con pino silvestre de su cuenca, con la finalidad de protegrer la carretera de Francia. Además, en última instancia se pretende, junto a las obras efectuadas en otros puntos de la cuenca del Gállego, regular el arrastre de sedimentos al vaso del embalse de La Peña, por aquellos años en construcción (DE LA RIVA FERNÁNDEZ, 1994, p. 475-476).

10 En las semanas posteriores a la tragedia se procedió a desescombrar parcialmente estas gradas, por lo que bastantes bloques fueron retirados.
} 
situación en que quedó el núcleo de Betés). Esta hipótesis se apoya en el hecho de que las dos gradas situadas aguas arriba de la confluencia de barrancos tan apenas han sufrido daños, en parte porque se hallan poco elevadas sobre el talweg, pero también porque recibieron caudales inferiores ${ }^{11}$. Por el contrario, las gradas situadas aguas abajo de esa confluencia están totalmente arrasadas. La vegetación que coloniza el fondo del barranco también señala una acusada diferencia en los procesos naturales: en las dos gradas superiores no ha resultado arrasada, aunque sí dañada; en las situadas más abajo, la denudación vegetal es prácticamente total.

El barranco de La Selva, que confluye en el de Arás enfrente del de Betés, tan apenas aportó material. Una pequeña presa de contención de sólidos y laminación de avenidas, ubicada cerca de su desembocadura, retuvo el escaso material movilizado aguas arriba (piedras de variado calibre y algunos árboles). El escaso volumen de este material incita a creer que sólo se vieron afectados bolos y cantos ubicados previamente sobre el cauce, sin que las laderas enmarcantes resultasen afectadas. La presa, construida en bloques de hormigón, ha resultado descalzada en su sector izquierdo, que se halla semitumbado.

La hipótesis del taponamiento también se fundamenta en otros datos:

- Pocos minutos antes de la riada que asoló el camping, y bajo una intensísima lluvia, testigos presenciales aseguran que tan apenas bajaba agua por el cauce del barranco. El arrasamiento del camping se produjo en pocos minutos, como consecuencia de una ola de agua repentina de gran volumen, que quedaría liberada tras la rotura del tapón.

- La absoluta destrucción de la gradas del tramo superior, provocada parcialmente por el choque de los grandes bloques morrénicos retenidos temporalmente y movilizados con gran violencia en el momento de la probable rotura del represamiento.

- Y, finalmente, la altura que alcanzaron las aguas -hasta $10 \mathrm{~m}$ sobre el fondo del cauce en puntos en los que éste supera los $25 \mathrm{~m}$ de ancho- no se explica únicamente por un aumento de caudal derivado de las precipitaciones.

Los efectos de la riada son diferentes en los dos tramos de la canalización de Arás antes delimitados. En el superior el arrasamiento es prácticamente total, habiendo desaparecido los diques de contención y las paredes laterales de

11 Estas gradas están aguas abajo, muy cerca, del puente de la carretera de acceso a Yosa, que sí fue derruido, al quedar descalzado, por socavamiento basal, uno de sus pilares. Pero hay que señalar que la solidez de las gradas es muy superior a la del puente. 
delimitación del canal. Unicamente se mantienen unos pocos muros de los que marcan el salto de las gradas. También han resultado muy afectados los sectores basales de las laderas enmarcantes, por erosión lateral, y una pista forestal que discurría por 1 a margen izquierda ha desparecido totalmente en bastantes sectores. En el tramo inferior de la canalización la destrucción es mucho menor. En general, 1 a infraestructura del canal se mantiene, aunque bastante dañada en muchos puntos y tapizada por los materiales heterométricos arrastrados.

Las razones que explican este diferente comportamiento de ambos tramos se van a intentar explicar a continuación.

En primer lugar, hay que hacer referencia a la distinta pendiente de uno y otro. Ya se ha indicado antes que la pendiente longitudinal del tramo superior es más alta, de manera que la energía de las aguas y de los probables materiales en movimiento liberados tras el supuesto represamiento es aquí mayor, por su mayor velocidad.

En segundo lugar, en el tramo superior el flujo discurrió encajado por la línea de talweg del valle. Pero en el tramo inferior se dividió en dos cauces diferentes: el del Arás canalizado y el antiguo cauce del barranco, a la derecha de la canalización, con los consiguientes efectos dispersores de energía. En la reutilización del cauce antiguo influyeron dos factores:

- El desbordamiento del canal, de manera que al llegar las aguas al punto superior del cono de deyección, aprovecharon la mayor amplitud topográfica para expandirse.

- La energía centrífuga del flujo, en el punto en que la canalización de Arás traza un curva a la derecha. La elevadísima velocidad del caudal favoreció el mantenimiento de la línea recta en el desplazamiento, retomando y ampliando por incisión lineal y erosión lateral el cauce natural, que en este tramo discurre a la izquierda de la canalización.

Este flujo inadaptado a la estructura del canal artificial chocó violentamente con la ladera enmarcante, que también dibuja una curva hacia la derecha. El "efecto rebote" consiguiente obligó al caudal a girar bruscamente, en ángulo próximo a los $90^{\circ}$, a la derecha, atravesando la canalización y adaptándose al viejo cauce. En este punto se produjo por lo tanto el desdoblamiento del flujo en dos cauces diferentes. E1 desbordamiento de ambos explica la catástrofe sufrida por el camping "Las Nieves".

Las características geométricas de la canalización de Arás no resultan las más adecuadas para desaguar, sin que se produzcan desbordamientos, caudales excepcionales, como los alcanzados en el evento que nos ocupa. Independientemente de 
que probablemente ningún diseño canalizador hubiera soportado sin problemas la evacuación del flujo en los momentos de mayores caudales, resulta conveniente comentar algunos fallos detectados.

- El sector superior de la canalización presenta una sección mayor que el inferior, como ya se ha comentado líneas arriba. Es más ancho y los muros laterales delimitadores, más altos. Puede, por lo tanto, admitir caudales superiores. Además, su mayor pendiente, que obliga al flujo a discurrir a más velocidad, es otro factor de su mayor capacidad evacuatoria. Ciertos volúmenes de caudales, que el sector superior podría evacuar, se desbordarían en el inferior $^{12}$.

- Los muros laterales delimitadores de la canalización no se hallan en toda su longitud a la misma altura relativa con respecto al fondo del canal. Inmediatamente antes de los saltos de las gradas, su altura es inferior a la existente después de dichos saltos. Dado que en condiciones de grandes caudales y elevada velocidad del flujo éste no se adapta a la forma escalonada de las gradas, sino que dibuja una lámina homogénea, en los puntos de menor altura de los muros se favorece el desbordamiento.

- La curva a la derecha que marca el contacto entre los dos sectores de la canalización es excesivamente brusca. Un cambio de sentido más suave y gradual dificultaría el desbordamiento por la tendencia del flujo a mantener la línea recta.

Uno de los sectores más interesantes para aproximarnos a la comprensión de los procesos geomorfológicos que explican la fortísima erosión en la cuenca del Arás y la caída de materiales al talweg, se halla situado en la margen derecha del valle, en el entorno de las denominadas "Señoritas de Arás". Aquí se ubica una morrena que favorece -por las características del depósito- el aporte de sedimentos, sobre la que se yerguen unas espectaculares dames coiffées (las "Señoritas de Arás"), aguas abajo del barranco de la Selva. Se trata de una morrena integrada por material heterométrico y cuyo estadio de evolución la convierte en un relieve muy inestable, buen ejemplo de lo cual es la presencia de las espectaculares columnas acabadas de citar. A ello se une la ausencia de cobertura vegetal y la existencia de fuertes pendientes (ver PELLICER CORELLANO, CANCER POMAR y ECHEVERRÍA ARNEDO, 1994). Las escorrentías en las vertientes provocadas por la precipitación han generado abundantes deslizamientos y algún derrumbe. El tramo inferior de la ladera, en contacto con el

12 Las obras de canalización del sector inferior de Arás fueron diseñadas para soportar un caudal máximo de $125 \mathrm{~m}^{3} / \mathrm{sg}$. Dado que su sección no es homogénea en toda su longitud, el volumen anterior debe ser considerado como dato aproximado. Considerando una anchura de $18 \mathrm{~m}$ y una altura media de $1,4 \mathrm{~m}$, la velocidad del flujo para alcanzar el antedicho caudal sería de $5 \mathrm{~m} / \mathrm{sg}$. 
talzveg, está socavado, por el efecto de erosión lateral del barranco de Arás. Los aportes de sedimentos de estos depósitos al cauce fueron, sin duda, muy elevados.

Tal como se ha explicado anteriormente, además de los depósitos morrénicos son muy abundantes los coluviales, sobre todo en el sector basal de las laderas. La presencia en el cauce del barranco de Arás de cantidades ingentes tanto de material morrénico como coluvial certifica que ambos tipos de depósitos actuaron de área fuente de los materiales movilizados.

Precisando algo más, se aprecia en estas áreas fuentes que al factor litogeomorfológico hay que sumarle el de ubicación a más o menos altura sobre el cauce. Allí donde los depósitos desprovistos de vegetación llegan hasta el fondo del valle, sí que se localizan abundantes deslizamientos y desplomes (por ejemplo, aguas arriba de las "Señoritas de Arás"). En general, estos márgenes están totalmente incididos por las aguas. Pero en los depósitos situados a cierta altitud, separados del talweg por una banda ancha vegetal, no se aprecia caída de materiales ni arrasamiento de la vegetación.

Respecto a los muy numerosos árboles y matorrales arrastrados por la crecida, proceden tanto del propio cauce (había pequeñas isletas habitualmente libres del paso de agua en donde creció la vegetación) como de los tramos inferiores de las laderas, totalmente afectados por la crecida.

Este comportamiento selectivo de los procesos erosivos, marcadamente diferente según los tramos de las vertientes incluso en episodios de elevadísima escorrentía superficial, indica que la colonización vegetal, a partir de la etapa de matorral denso, controla la escorrentía y la producción de sedimentos, que tiende a localizarse en los cauces fluviales y en sus proximidades (GARCÍA RUIZ, 1996).

La erosión generada en el cauce ha sido tanto lateral (muy importante, por el gran desbordamiento del barranco) como lineal, apreciándose en bastantes tramos in acusado encajamiento sobre los depósitos torrenciales que tapizan el fondo del valle.

La denudación vegetal en las áreas afectadas por la crecida ha sido de gran magnitud; se puede afirmar que el cauce del barranco y el sector basal de las laderas enmarcantes han quedado totalmente arrasados. Numerosos ejemplares de pinos silvestres, quejigos, bojes, sauces... fueron arrancados y transportados a considerable distancia. El tramo en que la transformación biogeográfica es más intensa coincide con el viejo cauce del Arás, reutilizado en esta crecida. Tras siete décadas en las que sólo ha llevado agua en momentos muy puntuales de desbordamiento del cauce canalizado, la vegetación lo había invadido totalmente, hasta el punto de que prácticamente pasaba desapercibido, a lo cual también contribuía su incisión lineal, poco marcada. 
Tras la crecida, la cobertura vegetal ha quedado arrasada y se dibuja un pasillo que indica perfectamente el sentido que llevaron las aguas.

\section{CONCLUSIONES}

La precipitación de más de $170 \mathrm{~mm} / \mathrm{m}^{2}$ en tan sólo 2,15 h., acaecida en la cuenca del barranco de Arás en la tarde del 7 de agosto de 1996, coincide con la esperada en la zona para un período de retorno ligeramente inferior a los 200 años. En condiciones de flujo normales, provocaría caudales de entre 330 y más de $900 \mathrm{~m}^{3} / \mathrm{sg}$, pero los aportes de sólidos al talweg -con abundantes bloques de calibre métrico-, sobre todo aguas abajo de la desembocadura del barranco de Betés, favorecieron probablemente la acumulación de materiales en los diques de las gradas superiores del tramo canalizado del barranco, y el "efecto barrera" consiguiente. Al romperse estos diques, se liberarían enormes volúmenes de agua y de sólidos que arrasaron en pocos minutos todo lo que encontraron su paso, también el camping "Las Nieves", situado en el cono de deyección del barranco.

En esta catástrofe no sólo influyeron los procesos climático-hidrológicos; también los geomorfológicos, derivados de la existencia de varias morrenas y de numerosos depósitos coluviales, fácilmente erosionables, que proveyeron de abundante material al barranco. El desbordamiento del cauce es un fenómeno natural en los cursos torrenciales, como el Arás, con consecuencias especialmente perniciosas en el cono de deyección, lugar en el que se situaba un camping objeto de un riesgo hidrológico evidente, aunque -con las características de la excepcional crecida del 7/8/1996- de muy baja frecuencia.

Aunque la magnitud de los procesos naturales está en la base de la catástrofe, determinadas modalidades de gestión del medio natural en la cuenca del barranco de Arás han contribuido a magnificar sus efectos.

\section{BIBLIOGRAFIA}

CANCER POMAR, L. (1995): Ecogeografia de los paisajes del Alto Gállego. Serie Investigación, 1, Publicaciones del Consejo de Protección de la Naturaleza de Aragón, Zaragoza.

CUADRAT PRATS, J.Ma (1981): El clima del Pirineo Central. Ensayo de aplicación al turismo de montaña. Departamento de Geografía y Ordenación del Territorio, Universidad de Zaragoza. (Tesis Doctoral inédita). 
DE LA RIVA FERNÁNDEZ, J. (1994): Los montes de la Jacetania: caracterización física y explotación. Departamento de Geografía y Ordenación del Territorio, Universidad de Zaragoza. (Tesis Doctoral inédita).

GARCÍA RUIZ, J. Ma (1996): "Marginación de tierras y erosión en áreas de montaña". En, T. Lasanta Martínez y J. M. García Ruiz (Eds.): Erosión y recuperación de tierras en áreas marginales, 33-50, Inst. de Estudios Riojanos-Sociedad Española de Geomorfología. Logroño.

GARCÍA RUIZ, J.Ma y PUIGDEFÁBREGAS, J. (1982): “Formas de erosión en el flysch eoceno surpirenaico". Cuadernos de Investigación Geográfica, VIII, 83-126. Colegio Universitario de Logroño.

GARCÍA RUIZ, J.M", PUIGDEFÁBREGAS, J. y CREUS, J. (1986): Los recursos hídricos superficiales del Alto Aragón. Instituto de Estudios Altoaragoneses, Diputación Provincial de Huesca.

GÓMEZ VILLAR, A. (1996): Conos aluviales en pequeñas cuencas torrenciales de montaña. Monografías Científicas, 6, Geoforma Ediciones, Logroño.

ICONA (1979): Precipitaciones máximas en España. Serie Monografías, 21. Madrid.

MARÍN JAIME, J.Ma (1987): Hidrología de la cuenca alta y media del río Gállego. Departamento de Geografía y Ordenación del Territorio, Universidad de Zaragoza. (Tesis Doctoral inédita).

MARTÍNEZ CASTROVIEJO, R. y GARCíA RUIZ, J.M" (1990): "Coladas de piedras (debris flows) y dinámica fluvial en ríos torrenciales del Pirineo Central: el caso del río ljuez". Cuadernos de Investigación Geográfica, 16, 55-72. Logroño.

PELLICER CORELLANO, F., CANCER POMAR, L. y ECHEVERRÍA ARNEDO, Ma T. (1994): "Las 'Señoritas de Arás', un elemento geomorfológico singular amenzado". Geographicalia, 31, 157-176, Dpto. de Geografía y Ordenación del Territorio, Universidad de Zaragoza.

SERRANO CANADAS, E. (1991): Geomorfología glaciar de las montañas y valles de Panticosa y de la Ribera de Biescas (Pirineo Aragonés). Univ. Autónoma de Madrid. (Tesis Doctoral). 


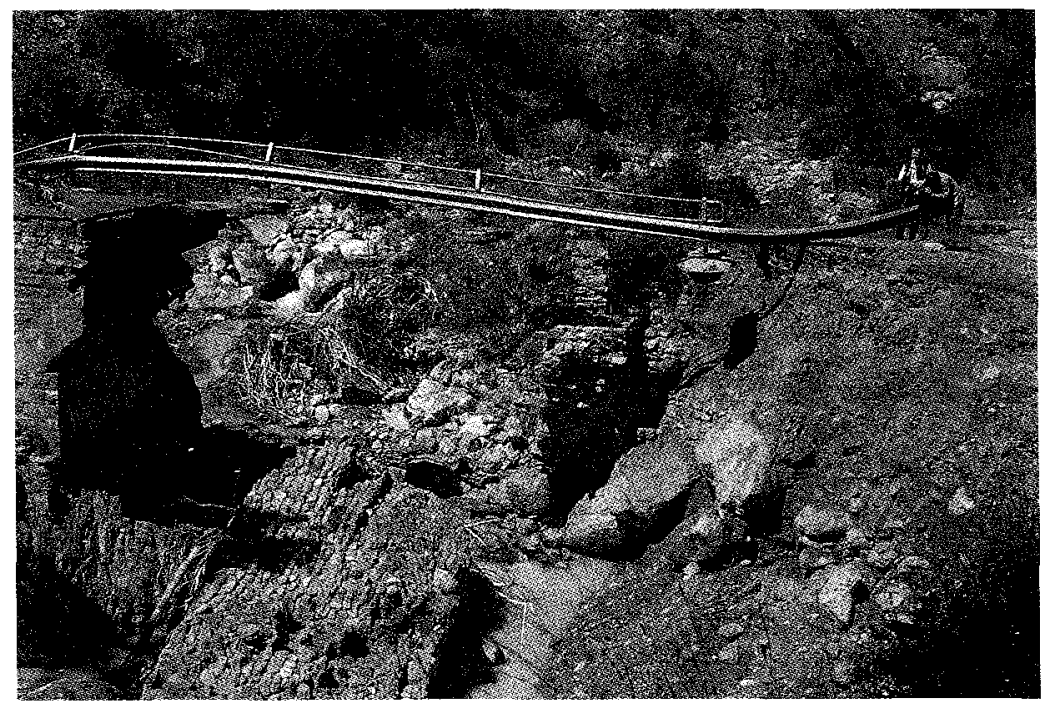

Fotografía 1.- Puente de Yosa, derruido por socavamiento basal de uno de sus pilares, que aparece tumbado en primer plano.

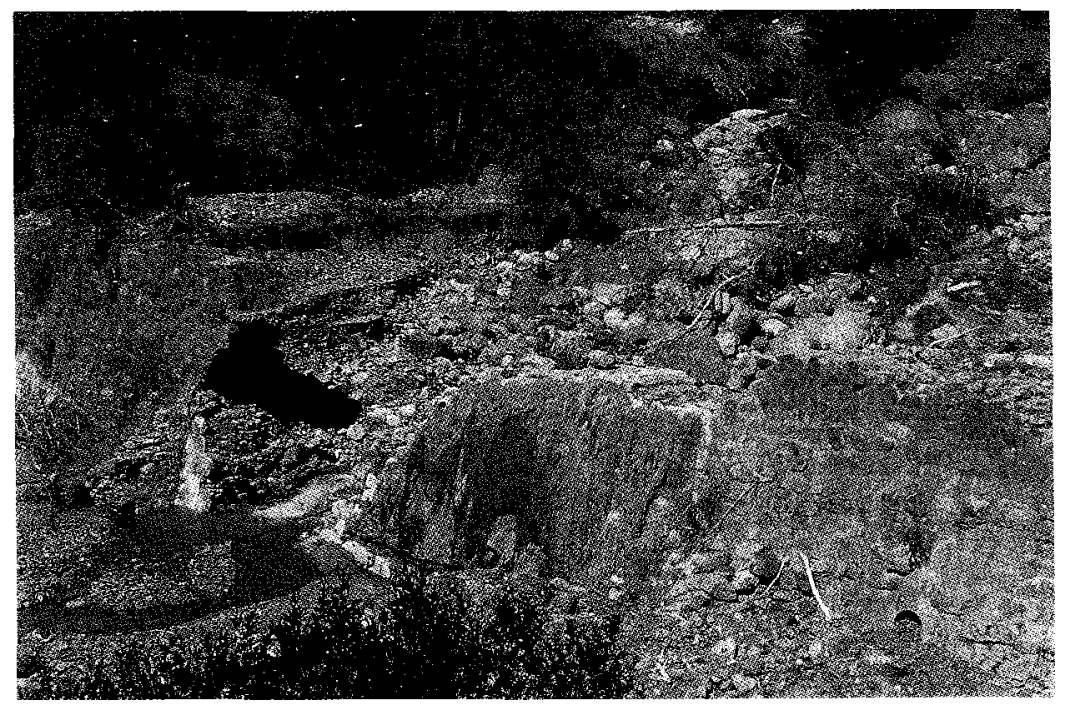

Fotografía 2.- Detalle de la destrucción de uno de los muros transversales de retención de sólidos y laminación de avenidas, en la parte alta de Arás. 


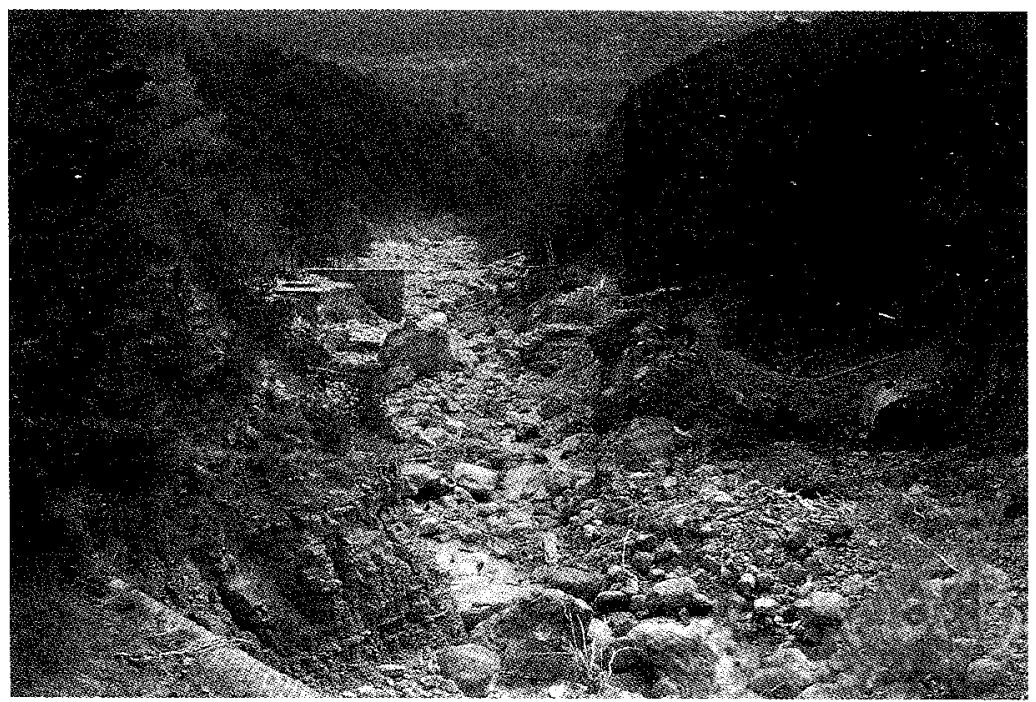

Fotografía 3.- Vista general del sector superior del barranco de Arás tras la riada. Apréciese la desaparición casi total de los muros laterales y transversales de la canalización, y la gran cantidad de materiales morrénicos y coluviales arrastrados y removidos por el flujo.

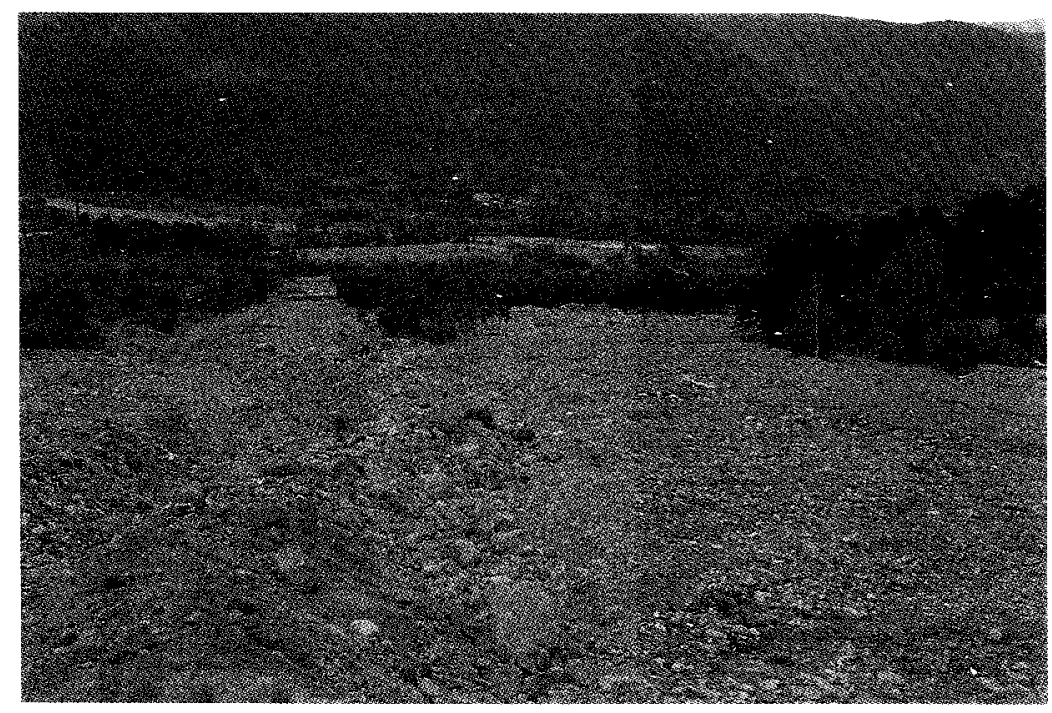

Fotografía 4.- Sector inferior de Arás, en el cono de deyección del barranco. A la izquierda, el tramo canalizado, rectilíneo y semitapizado por los sedimentos aportados por la crecida. A la derecha, el antiguo cauce -cauce natural-, reutilizado por el flujo. Entre ambos se construyó el camping "Las Nieves". 